\title{
Optimal Placement and Sizing of Distributed Generation Using Metaheuristic Algorithm
}

\author{
D. Nageswari ${ }^{1, *}$, N. Kalaiarasi ${ }^{2}$ and G. Geethamahalakshmi ${ }^{1}$ \\ ${ }^{1}$ Department of Electrical and Electronics Engineering, RMK College of Engineering and Technology (RMKCET), Thiruvallur, \\ 601206, India \\ ${ }^{2}$ Department of Electrical and Electronics Engineering, RMK Engineering College, Gummidipoondi, 601206, India \\ *Corresponding Author: D. Nageswari. Email: nageswariraghava@gmail.com \\ Received: 28 May 2021; Accepted: 02 July 2021
}

\begin{abstract}
Power loss and voltage uncertainty are the major issues prevalently faced in the design of distribution systems. But such issues can be resolved through effective usage of networking reconfiguration that has a combination of Distributed Generation (DG) units from distribution networks. In this point of view, optimal placement and sizing of DGs are effective ways to boost the performance of power systems. The optimum allocation of DGs resolves various problems namely, power loss, voltage profile improvement, enhanced reliability, system stability, and performance. Several research works have been conducted to address the distribution system problems in terms of power loss, energy loss, voltage profile, and voltage stability depending upon optimal DG distribution. With this motivation, the current study designs a Chaotic Artificial Flora Optimization based on Optimal Placement and Sizing of DGs (CAFO-OPSDG) to enhance the voltage profiles and mitigate the power loss. Besides, the CAFO algorithm is derived from the incorporation of chaos theory concept into conventional artificial flora optimization AFO algorithm with an aim to enhance the global optimization abilities. The fitness function of CAFO-OPSDG algorithm involves voltage regulation, power loss minimization, and penalty cost. To consider the actual power system scenario, the penalty factor acts as an important element not only to minimize the total power loss but to increase the voltage profiles as well. The experimental validation of the CAFO-OPSDG algorithm was conducted against IEEE 33 Bus system and IEEE 69 Bus system. The outcomes were examined under various test scenarios. The results of the experiment established that the presented CAFO-OPSDG model is effective in terms of reducing the power loss and voltage deviation and boost-up the voltage profile for the specified system.
\end{abstract}

Keywords: Voltage profile enhancement; loss minimization; distributed generation; optimal dg sizing; optimal DG placement; metaheuristics

This work is licensed under a Creative Commons Attribution 4.0 International License, which permits unrestricted use, distribution, and reproduction in any medium, provided the original work is properly cited. 


\section{Introduction}

Electricity plays a major role in sustainable growth and development of a country, especially its economy. It exerts a considerable impact and impact the human lives. Several researchers have developed efficient techniques to generate electricity in an optimum and inexpensive manner. In most of the cases, the electricity generation plants are situated away from the consumers. So, the power produced in these generation plants needs to be transmitted via high-voltage transmission systems to distribution systems and finally reaches the end-users i.e., consumers with no disturbance and loss. However, in practical, the power generated in the power plants does not reach the consumer in its full capacity, owing to the generation loss, transmission loss, etc [1]. A considerable amount of power is lost at the distribution level over Power Loss (PL) that occurs in the stages of production and transmission. A considerable PL is incurred due to the fact that the voltage at distribution stage is minimum and current is maximum. In addition, the resistivity of the distribution line is highly significant over transmission line resistance counterpart.

Electrical distribution companies face crucial issues with regards to PL and voltage instability. The utilities pay significant attention to system reliability, voltage regulation, active power $(\mathrm{P})$ and reactive power (Q) issues, because of the maximum penetration level of alternative Renewable Energy Sources (RES) in distribution network. So, the Distributed Generation (DG) unit is estimated to track severe technological and regulatory necessities to guarantee security, steadiness, and effectiveness of the distribution networks [2]. The present distribution network faces a number of challenging issues. Voltage profile is highly important for the consumer, since quality voltage-controlled electrical equipment demand quality power to be supplied. DGs can offer voltage support to raise the voltage levels. The major benefits of DG in power system are better power stability, improved distribution network reliability and operational and cost-effective aids. The advantages of utility and user comprise of enhanced voltage profile, low PL, peak load shaving, reduced transmission, and extension of distribution network [3]. But, when the number of DG units are abundant in network, it might lead to numerous issues like system protection, overvoltage, under-voltage, reverse power flow, power quality problem, overloading, and thermal overloading of the distribution line.

Next to deregulation of power systems, several power companies have started investing in small-scale RESs like wind, photovoltaic cells, micro-turbine, small hydro-turbine etc., to fulfill the power needs and make profit. The presence of DG units in power systems can considerably decrease the PL. But the level of DG penetration vs. PL seems to be a U-trajectory. So, the non-optimal placements of DG might raise the PL and decrease the voltage profiles to a maximum extent [4,5]. The placement of optimal DGs and sizing techniques are mandatory to reduce the system loss and increase the voltage profile. Several works conducted earlier have proposed designs for integrating DGs and power system in order to mitigate the PLs, enhance power efficiency, reduce line current, boost voltage profile and increase system stability and load efficiency. Diverse techniques and optimization algorithms have been employed in the integration of DG units with power systems [6]. The researchers have examined diverse techniques to place DGs in a radial distribution system by following methods that depend on heuristics and metaheuristics. Furthermore, the placement and DG sizing are defined using nature-inspired algorithms such as Genetic Algorithm (GA) and Whale Optimization Algorithm (WOA) etc., [7,8].

In this view, the current study designs a Chaotic Artificial Flora Optimization based on Optimal Placement and Sizing of DGs (CAFO-OPSDG) to improve the voltage profiles and mitigate power loss. The aim of this study is to determine the optimum location and sizing of DGs that can considerably reduce the PL and improve voltage stability. The proposed CAFO-OPSDG technique derives a fitness function that is comprised of voltage regulation, PL minimization, and a penalty cost. In addition, the CAFO algorithm incorporates the concept of chaos theory to improve the global optimization capabilities of traditional AFO algorithm. The proposed CAFO-OPSDG algorithm was experimentally validated 
against IEEE 33 Bus system and IEEE 69 Bus system and the results were examined with respect to reduction in PLs and voltage deviation, and boost-up the system voltage profile. In short, the contribution of this paper is as follows.

- A novel CAFO technique is developed for optimal placement and sizing of DGs

- The goal of CAFO-OPSDG technique is to decrease the PL and increase the voltage profiles

- The CAFO algorithm is designed by the integration of AFO algorithm with chaos theory in such a way that global optimization capability of the AFO algorithm is increased

- A fitness function is derived that involves voltage regulation, PL minimization, and penalty cost

- The performance of the CAFO-OPSDG technique is validated on standard IEEE 33 Bus system and IEEE 69 Bus system.

Rest of the paper is organized as follows. Section 2 reviews the recent state-of-the-art methods related to optimum sizing and placement of DGs. Section 3 discusses the presented CAFO-OPSDG technique and Section 4 validates the performance under diverse aspects. At last, Section 5 concludes the paper.

\section{Literature Survey}

The current section reviews the recently-presented techniques for optimum placement and sizing of DGs to mitigate the losses and enhance the voltage profiles. In Naderipour et al. [9], several kinds of loads including constant current, impedance, and power are deliberated. The study resolved these with the help of Spotted Hyena Optimizer ( $\mathrm{SHO}$ ) model to determine an optimal location and capacitor size, assume distinct loading levels and two operation modes. To evaluate the efficiency of SHO, the outcomes were compared with GWO and Genetic Algorithm (GA) techniques. A research study was conducted by Haider et al. [10] for optimal location and sizing of the DG to a radial distributed network after and before the re-configuration. A multi-objective PSO method was used in this study to determine the optimum deployment and sizing of DGs after and before re-configuration. An optimum network structure with DG coordination for active network resolves the PL, enhances the system stability and efficiency, uplifts voltage profiles and reliability. A penalty factor is assumed to consider the real power system situations. It acts as a critical part in overall PL and voltage profile enhancement.

Nguyen et al. [11] proposed a reconfiguration method based on CSA to mitigate active PL and maximize the voltage magnitude. This technique is a novel metaheuristic technique, stimulated from the required brood parasitism of few cuckoo species. These birds place their eggs in the nest of other host bird species. The same pattern was followed in this technique to resolve the optimization problem. In Kasi et al. [12], Harris hawks optimization technique was utilized to resolve the DG deployment problem in microgrid. It efficiently moderates the actual PL and enhance the voltage profile. Therefore, it reduces the real PL with the help of FF to place DG units and size it in the microgrid. The proposed technique was employed in IEEE investigation schemes such as 33 and 69 bus microgrids. Essallah et al. [13] presented MPSO method for active PL reduction and voltage profile enhancement from distributed networks. This method connects traditional PSO and BPSO methods. Initially, it is dedicated to identify the optimum distributed networks, when the additional one is utilized to resolve the DG deployment and sizing challenges. Dash et al. [14] attempted to achieve the minimization of communication loss in IEEE network via effective deployment of FACTS device. The major goal was attained by minimizing the installation cost of this device as assumed by FF for optimization method. In this study, MFO was employed in its hybrid and natural forms named JAYA blended MFO (JMFO).

In Ramamoorthy et al. [15], an integrated nature-inspired method was presented to optimize the location and sizing of DGs. The study recommended a 2-stage optimization method to combine DG units. Initially, the optimum size of DG was defined by PSO metaheuristics and the outcomes were attained by PSO. 
These were then established to reverse the power flow through negative load method so as to find the probable bus location. Later, an optimum location is created by LSF and WK bus approaches with related outcomes. Next, the optimum sizing of DGs is defined with the help of hybrid PSOGSA, GSA, and PSO methods. Yenealem et al. [16] aimed at combining micro grid scheme with STATCOM (static synchronous compensator) controller to ensure high power flow through improved voltage profile and decreased PL.

In Ziari et al. [17], altered distinct PSO was proposed to detect an optimal deployment and sizing of capacitor in a distributed scheme. The decision criteria consist of line loss and capacitor investment cost. Both feeder current and bus voltage limitations are involved in decision criterion via limited penalty factors. In Devabalaji et al. [18], a novel model was examined to determine an optimum location and sizing of DSTATCOM and DG, and the decision criterion was expressed to minimize the voltage profile, operation cost, and PL improvement of the scheme exposed to equality and equality limitations. LSF is utilized to predetermine the optimum location of DSTATCOM and DG. BFOA method is presented to determine the optimum size of DSTATCOM and DG. Hassan et al. [19] utilized BPSO and SLFA techniques to simulate 33 and 69 bus radial distributed schemes. The experimental outcome demonstrated the optimal distribution of DGs and mitigation of PL. Few research works have also been conducted on hybridization of Particle Swarm Optimization (PSO) and Genetic Algorithm (GA) for optimal location and placement of DGs in order to reduce power loss and enhance voltage profiles. The PSO-GA model was tested using standard 29 bus system and the results were validated against different cases. Though the PSO-GA model achieved effective performance, there is a need still exists to derive a novel technique to design the PSO-GA technique to enhance the voltage profiles and reduce the power loss.

\section{The Proposed Model}

\subsection{Problem Formulation}

Minimization of PL, stabilizing the bus voltage, and maximization of voltage stabilities are regarded as the major criteria for optimum placement and sizing of GD units. Electricity is generated at a location far away from the consumer and is distributed among the broadcast lines to several distribution systems in which the power utility operates. Usually, the distribution network system receives the power and transmits it to the consumer to satisfy the service level agreement. The total power is not received by the consumer owing to the loss incurred in distribution network line, which is mainly based on the proper placement and sizing of DG units. The actual PL in distribution systems, with a predefined operational setting, is determined with the help of Eq. (1) and is mentioned as an exact loss.

Minimize Ploss $(F 1)=\sum_{i=1}^{n b r} \mathrm{I} i^{2} R i$

where I $i$ and $R i$ represent the current magnitude and resistance equivalent to circuit branch I whereas the branch count is denoted by sd $b r$. Branch circuit current can be partitioned into two elements namely, active components (Iac) and reactive components (Irc).

$P L a(F 2)=\sum_{i=1}^{\mathrm{n}} \mathrm{I}^{2} a c i R i$

$\operatorname{PLr}(F 3)=\sum_{i=1}^{\mathrm{n}} \mathrm{I}^{2} r c i R i$

But the optimum placement and sizing of DGs compensate the active loss elements that exist in the branch. 
$P G=P D+$ Losses

where PG and PD indicate the real generator power and demand power. For voltage limit, Eq. (5), is used to enhance the voltage profiles as a major objective.

$F 4=\sum_{N i=1}^{N N}\left(V_{N \mathrm{i}}-V_{\text {rated }}\right)$

\subsection{Algorithmic Design of CAFO}

AFO is a state-of-the-art SI-based optimization method presented by Cheng et al. [20] and it depends upon flora migration and reproduction procedure. It is established on six standard operations and demonstrates powerful exploration capability with fast convergence speed. In this method, the original plants are generated primarily whereas the scattered seeds are known as offspring plants that are located at a specific distance. Initially, the early population is created arbitrarily with $N$ original plants.

$X_{i, j}=r \times D \times 2-D$

where the original plant position is represented with $X_{i, j}$. Here, $i$ and $j$ denote the dimensions and number of plants correspondingly, and $r$ indicates the uniform distribution in the range of $[0,1]$.

Then, the propagate distance $D_{j}$ is evaluated for all the plants. The evaluation of propagation distance of plant is based on two prior generations.

$D_{j}=D_{1 j} \times r \times c_{1}+D_{2 j} \times r \times c_{2}$

where propagation distances of the grandparent and parent plants are denoted by $D_{2 j}$ and $D_{1 j}$, correspondingly, $c_{1}$ and $c_{2}$ denote the learning coefficient, and $r$ indicates the uniform distribution. A novel grandparent propagation is given by

$D_{1 j}^{\prime}=D_{2 j}$

The novel parent spreading distance is given by [21]:

$D_{2 j}^{\prime}=\sqrt{\frac{\sum_{i=1}^{N}\left(X_{i j}-X_{i j}^{\prime}\right)^{2}}{N}}$

The offspring plant locations, near the original plant, are estimated as given herewith.

$X_{i, j \times m}^{\prime}=R_{i j \times m}+X_{i, j}$

where $X_{i j}^{\prime}$ represents the offspring plant locations, $m$ indicates the amount of off springs created with single plant, and $R_{i, j \times m}$ denotes the normal distribution arbitrary amount by mean zero and variance $D_{j}$. Few created offspring would survive whereas others do not. Either an offspring is alive/not can be defined by the likelihood of survival, which is estimated with the help of proportion-based selection.

$p=\left|\sqrt{\frac{F\left(X_{i j \times m}^{\prime}\right)}{F_{\max }}}\right| \times Q^{(j \dot{\times} m-1)}$ 
where the chosen likelihood is represented as $Q^{(j \times m-1)}$ and its value is between zero and one, $F_{\max }$ represents the maximum fitness of overall offspring, and every single offspring plant is denoted by $F\left(X_{i, j \times m}^{\prime}\right)$. The offspring tend to survive, when the likelihood of surviving $p$ is higher compared to $r$, in which $r$ denotes the uniform distribution arbitrary amount in interval zero and one. The $N$ offspring plant is chosen by the survived offspring as novel original plant for succeeding iteration. AF method is repeated till it encounters the end condition. At last, an optimum solution is selected. Fig. 1 showcases the flowchart of AFO technique.

Algorithm 1: Pseudocode of AF algorithm

Initiation: Generate $N$ original plant arbitrarily by Eq. (6); calculate fitness of every individual; select an optimum solution

while $t<$ MaxIter do

for $i$ from 1 to $N * M$ do

Evaluate the proportion distance by Eqs. (7)-(9)

Create offspring plant by Eq. (10)

if $p>r$ then

Offspring plant live

else

Offspring plant not live

end if

end for

Calculate novel solution

Arbitrarily select $\mathrm{N}$ novel original plant

The novel solution replace the older one when the value is optimum

end while

return an optimum solutions

To enhance the global optimization ability of AFO technique, chaos concept is included. Chaos is an unbalanced state which is highly sensitive to initial condition. It can be employed in several optimization algorithms to prevent the local optima problems and enhance the quality of solution. Since metaheuristic algorithm depends upon two phases namely, exploitation and exploration, chaos concept is introduced to maintain an effective trade-off between exploration and exploitation thereby achieving the optimum solution effectively [22].

In AFO, the parameter $\theta$ is defined as an important one since it affects the convergence rate of AFO technique. The performance of AFO technique is highly dependent upon their parameters. It can be noticed in Eq. (12) which suggests a vital momentum to start with the help of feasible searching space and it could not be exposed effectually. Chaos is employed to attain the enhanced characteristics in both exploration and exploitation in all the searching regions. This is done to improve the results for identifying the optimal global solutions. Chaotic maps are utilized to define the location $x_{i}^{k}$, where the 
variable $\theta$ is replaced with a value achieved with chaotic map as provided herewith.

$x_{i}^{k+1}=x_{i}^{k}+C_{\text {nap }} \times\left(x_{B H}-x_{i}^{k}\right), i=1,2, \ldots, N_{\nabla}$

where $x_{i}^{k}$ and $x_{i}^{k+1}$ denote the place of $\mathrm{i}^{\text {th }}$ star at rounds, $k$ and $k+1$ correspondingly. $x_{B H}$ implies the position of BH from space, $C_{\text {map }}$ refers to chaotic map, and $N_{s}$ signifies the star count. On the whole, 10 chaotic maps are utilized to manipulate the values of arbitrary parameters from AFO technique.

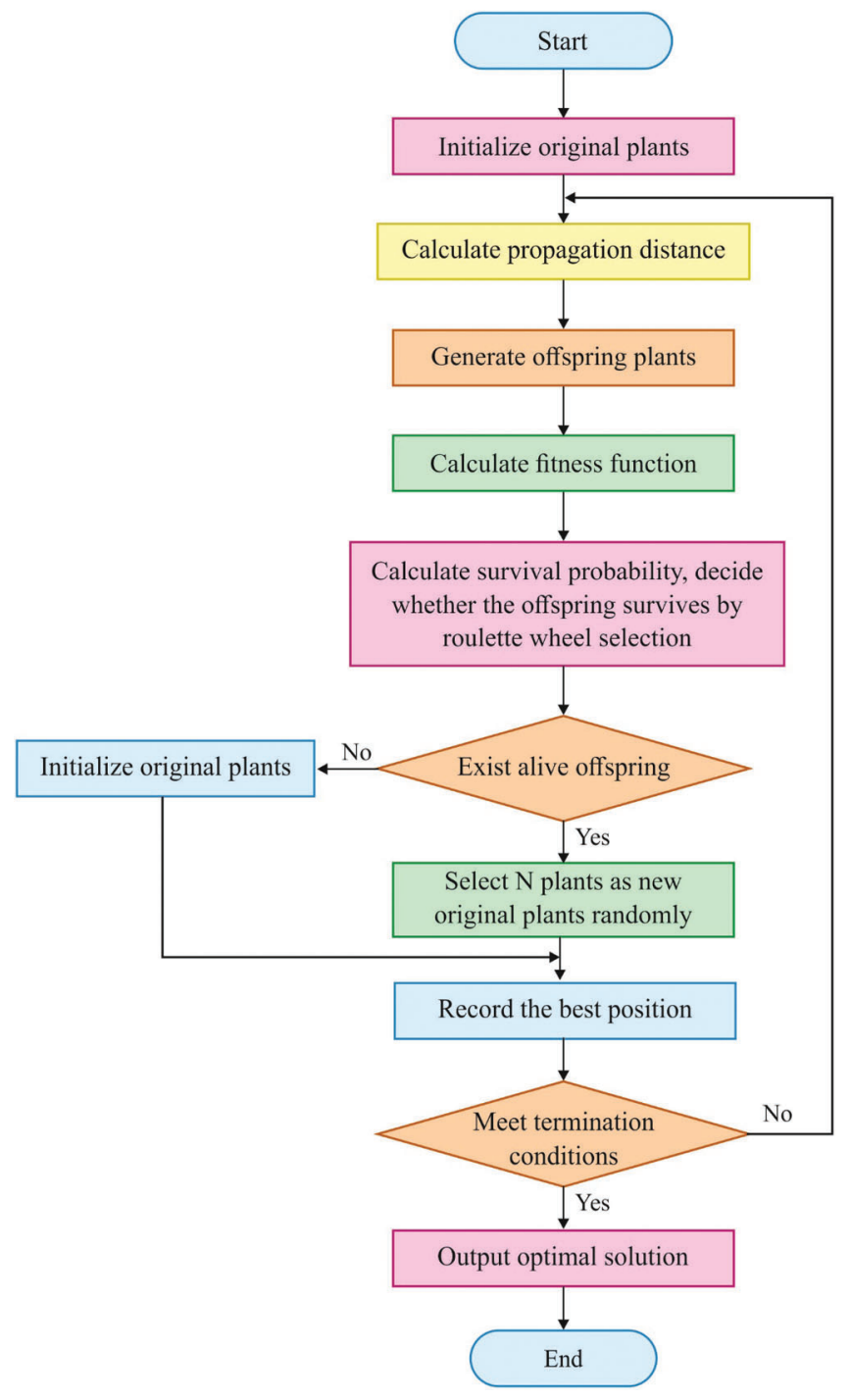

Figure 1: Flowchart of AFO

\subsection{Process Involved in CAFO-Based Optimal Placement and Sizing of DGs}

Fig. 2 demonstrates the overall working process of the proposed CAFO-OPSDG method. 


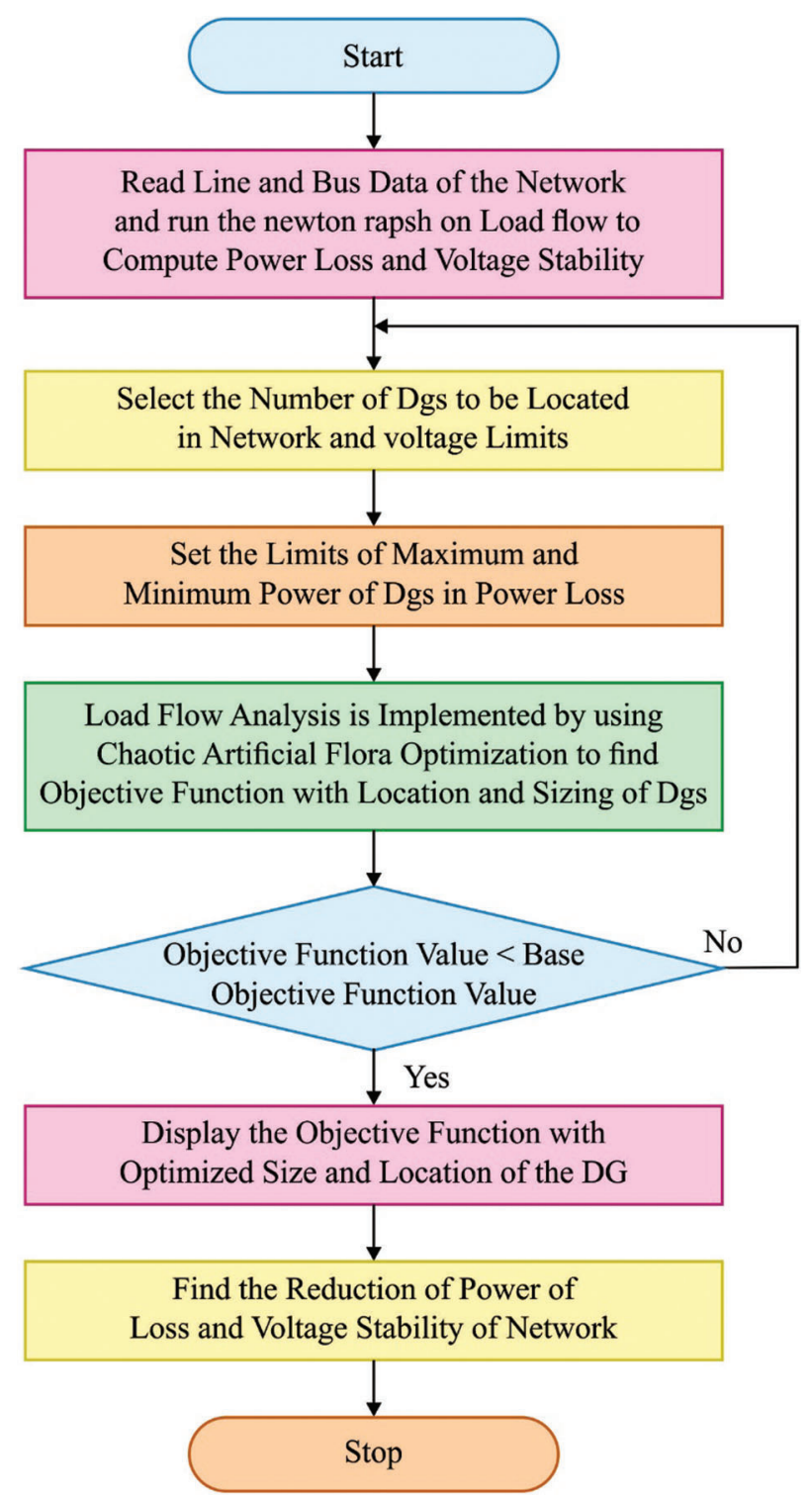

Figure 2: Steps followed in proposed model

CAFO-OPSDG technique is developed to concentrate on voltage regulation, PL reduction, and penalty cost. The violation of voltage limitation and total PL are involved in the derivation of FF. The penalty factor forces the voltage from violating the bounds so as to stabilize the voltage and minimize the PL of system. CAFO technique is used to optimally place the DGs and size it using the objective function given in Eq. (13).

$\min \mathrm{F}=\sum_{z}^{n} C_{z}^{D G} \mu_{z}+\sum_{z}^{n} \sum_{z+1}^{n} C_{(z, z+1)}^{\text {loss }} S_{(z, z+1)}^{\text {loss }}+\sum_{z}^{n} C_{z}^{\text {viol }} V_{z}$

The penalty cost to violate the voltage limit is found to be high, over the penalty cost of total PL, as defined in Eq. (14). So, the voltage values are precise and highly effective. The aim of the CAFO-OPSDG technique is to mitigate the overall DG placement cost $\left(C^{D G}\right)$, penalty cost for total $\mathrm{PL}\left(C^{\text {loss }}\right)$, and penalty cost in violating the limit in distribution system $\left(C^{\text {viol }}\right)$. 
$C^{\text {viol }}>C_{(z, z+1)}^{\text {loss }}$

DGs acts as a vital part in the conversion of conventional distribution network into active ones [23]. The conversion of a conventional radial distribution system into an independent microgrid network includes the optimal placement and sizing of DGs. Concurrently, the factors that need to be fulfilled are operating limitations to minimize the network PL, controlling the voltage deviation in every bus (with predefined limits), current capacity of the feeder, and voltage profile of systems. If DG units are allotted to bus $\mathrm{k}$ in the form of a binary variable $\mu_{z}$, its value can be considered as 1 , else 0 , as represented using Eq. (15).

$\mu_{z}=\left\{\begin{array}{l}1, \text { if DG is integrated at bus } \mathrm{z} \\ 0, \text { otherwise }\end{array}\right.$

The voltage variance at every bus needs to lie in maximum and minimum limits in order to ensure voltage stability and power quality. The voltage limits are measured in the range of $\pm 5 \%$ of the rated voltage for the load bus, as given below:

$V^{\min } \leq V_{z} \leq V^{\max }$

where, $V_{z}$ denotes the $V^{\text {th }}$ node voltage, and $V^{\text {min }}$ and $V^{\max }$ denote the lower and upper voltage bounds. The voltage drops per unit are determined with the help of Eq. (17) altered as Eq. (18) in case of injecting DG at node $z$, whereas $\mathrm{R}$ and $\mathrm{X}$ are resistance and reactance of line linking bus $z$ and bus $z+1$.

$V_{z}-V_{z+1}=P_{(z, z+1)} R_{(z, z+1)}+Q_{(z, z+1)} X_{(z, z+1)}$

$V_{z}-V_{z+1}=\left(P_{z+1}^{\text {load }}-P_{D G z+1}\right) R_{(z, z+1)}+\left(Q_{z+1}^{\text {load }}-Q_{D G z+1}\right) X_{(z, z+1)}$

The entire active PL $P_{(z, z+1)}^{\text {loss }}$ and reactive PL $Q_{(z, z+1)}^{\text {loss }}$ can be determined by Eqs. (19) and (20), The entire $\mathrm{PL}$ of network $S_{(z, z+1)}^{\text {loss }}$ is signified as the sum of $P_{(z, z+1)}^{\text {loss }}$ and $Q_{(z, z+1)}^{\text {loss }}$.

$P_{(z, z+1)}^{\text {loss }}=\sum_{z=1}^{n} \sum_{z+1=1}^{n}\left(\alpha_{(z, z+1)}\left(P_{z} P_{z+1}+Q_{z} Q_{z+1}\right)+\beta_{(z, z+1)}\left(Q_{z} P_{z+1}-P_{z} Q_{z+1}\right)\right)$

$Q_{(z, z+1)}^{\text {loss }}=\sum_{z=1}^{n} \sum_{z+1=1}^{n}\left(\gamma_{(z, z+1)}\left(P_{z} P_{z+1}+Q_{z} Q_{z+1}\right)+\delta_{(z, z+1)}\left(Q_{z} P_{z+1}-P_{z} Q_{z+1}\right)\right)$

$S_{(z, z+1)}^{\text {loss }}=P_{(z, z+1)}^{\text {loss }}+j Q_{(z, z+1)}^{\text {loss }}$

Each branch needs to encompass a flow of current below the thermal permissible bounds, as given herewith.

$I_{z} \leq I^{\max }$

where $I_{z}$ represents the $z^{\text {th }}$ branch current, and $I^{\text {max }}$ indicates the maximum current flow bound.

\section{Performance Validation}

This section validates the efficiency improvement of the presented model on two standard distribution networks such as IEEE 33 Bus system and IEEE 69 Bus system with base voltage of $12.66 \mathrm{kV}$. The line diagram of two bus systems is shown in Figs. 3 and 4. 


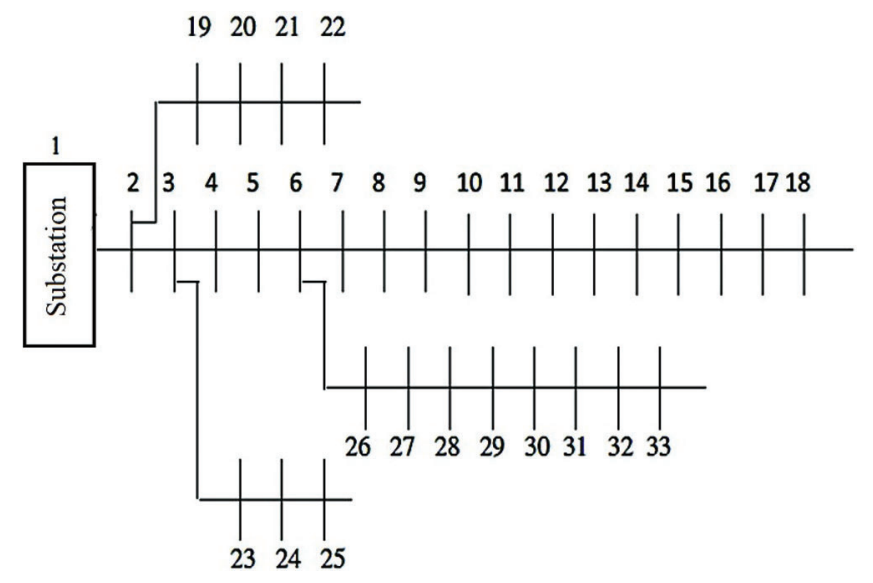

Figure 3: IEEE 33 bus system

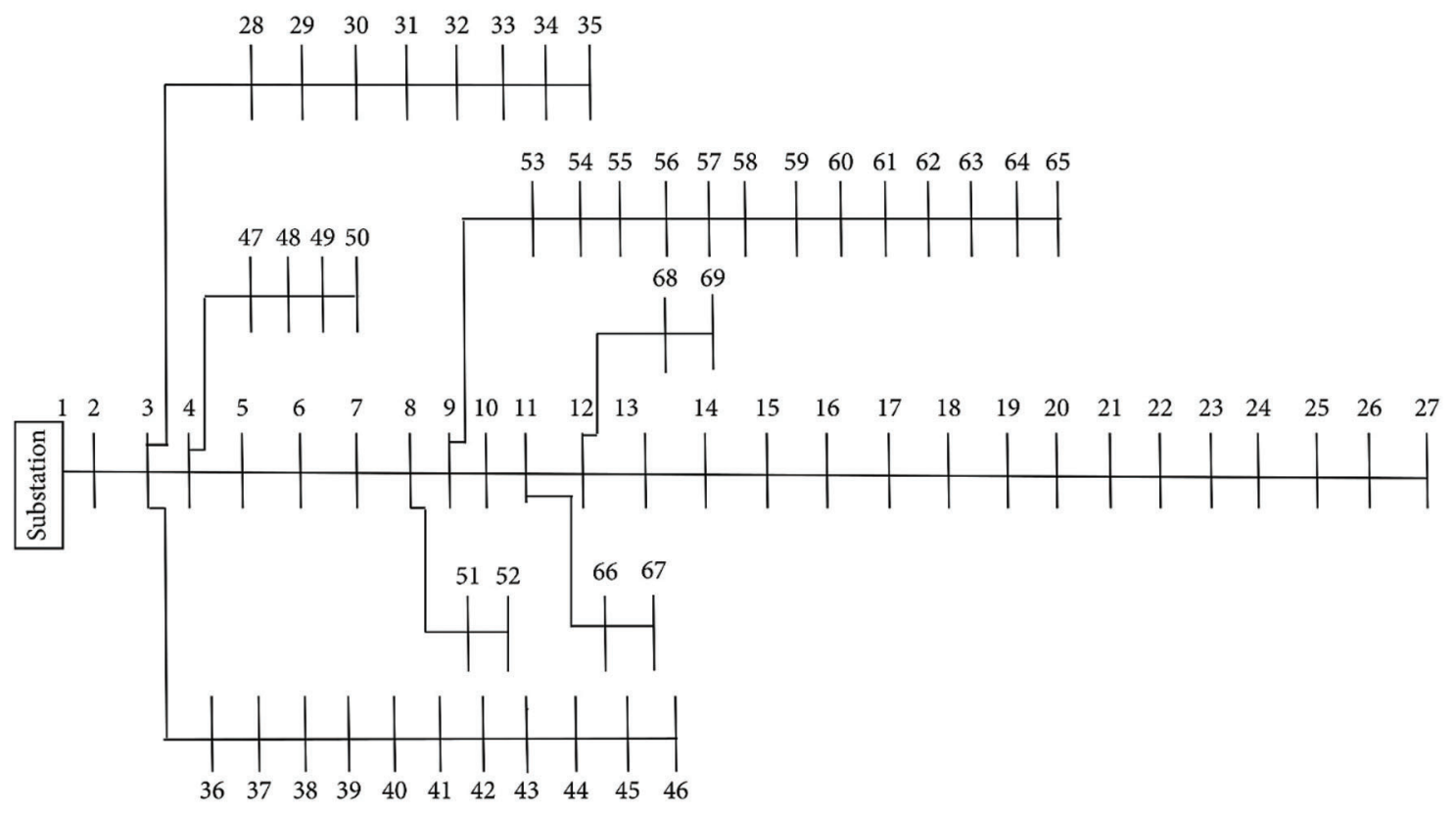

Figure 4: IEEE 69 bus system

Besides, the results were examined under four distinct scenarios, as listed below.

Case 1: Reconfiguration only

The reconfiguration setting of the distribution system occurs with the help of the proposed model to handle the functioning of switches. The proposed model was employed to resolve the issues related to the choice of sectionalizing the switches between the two possible states namely, open and close. The open and closed levels of the switch are represented using two criteria namely, 0 and 1.

Case 2: DG units only

It includes a series of many DG units (upto 3DGs) from bus system with no reconfiguration.

Case 3: Single DG unit reconfiguration and installation 
Two distinct levels are involved. At the first level, the reconfiguration takes place in the preferable bus system. In the second level, single DG unit is linked with reconfigured bus system.

Case 4: Multi DG units reconfiguration and installation

Reconfiguration takes place on the bus system. Many DG units (upto 3DG) are linked to the reconfigured bus systems such as standard 33 and 69 IEEE bus system.

A comprehensive results of the analysis of CAFO-OPSDG technique on IEEE 33 bus system at different scenarios are provided in Tab. 1 and Figs. 5 and 6. The table values demonstrate that the proposed CAFOOPSDG technique accomplished a minimal PL on all the applied cases. For instance, in case 1, the CAFOOPSDG technique reduced the PL from $202.68 \mathrm{~kW}$ to $72.6790 \mathrm{~kW}$ i.e., $64.1410 \%$ reduction in PL. At the same time, the CAFO-OPSDG technique achieved a minimal PL from $202.68 \mathrm{~kW}$ into $56.2893 \mathrm{~kW}$ i.e., $72.2275 \%$ reduction in PL. Moreover, in case 3, the CAFO-OPSDG technique accomplished the least PL from $202.68 \mathrm{~kW}$ to $44.8965 \mathrm{~kW}$ i.e., $77.8486 \%$ reduction in PL. Furthermore, the CAFO-OPSDG technique attained the least PL from $202.68 \mathrm{~kW}$ to $26.8629 \mathrm{~kW}$, i.e., $86.7461 \%$ reduction in PL. From these values, it is evident that the proposed CAFO-OPSDG technique has accomplished the least PL.

Table 1: Comparison of the proposed CAFO-OPSDG technique on IEEE 33 bus system at different scenarios

\begin{tabular}{lll}
\hline Case 1 & Before DG installation & After DG installation \\
\hline PL & $202.68 \mathrm{~kW}$ & $72.6790 \mathrm{~kW}$ \\
\% Reduction in PL & - & 64.1410 \\
Minimized voltage (pu) & 0.91075 & 0.93145 \\
Case 2 & & \\
PL & $202.68 \mathrm{~kW}$ & $56.2893 \mathrm{~kW}$ \\
\% Reduction in PL & - & 72.2275 \\
Minimized voltage (pu) & 0.91075 & 0.92945 \\
Case 3 & & \\
PL & $202.68 \mathrm{~kW}$ & $44.8965 \mathrm{~kW}$ \\
\% Reduction in PL & - & 77.8486 \\
Minimized voltage (pu) & 0.91075 & 0.92514 \\
Case 4 & & \\
PL & $202.68 \mathrm{~kW}$ & $26.8629 \mathrm{~kW}$ \\
\% Reduction in PL & - & 86.7461 \\
Minimized voltage (pu) & 0.91075 & 0.96541 \\
\hline
\end{tabular}

On the other hand, when examining the performance in terms of minimized voltage, the CAFO-OPSDG technique accomplished a high minimized voltage. For instance, under case 1, the CAFO-OPSDG technique achieved a minimized voltage of $0.93145 \mathrm{pu}$. Eventually, under case 2, the CAFO-OPSDG technique attained a minimized voltage of $0.92945 \mathrm{pu}$. Meanwhile, under case 3, the CAFO-OPSDG approach obtained a minimized voltage of $0.92514 \mathrm{pu}$. At last, under case 4, the CAFO-OPSDG method reached a minimized voltage of $0.96541 \mathrm{pu}$. 


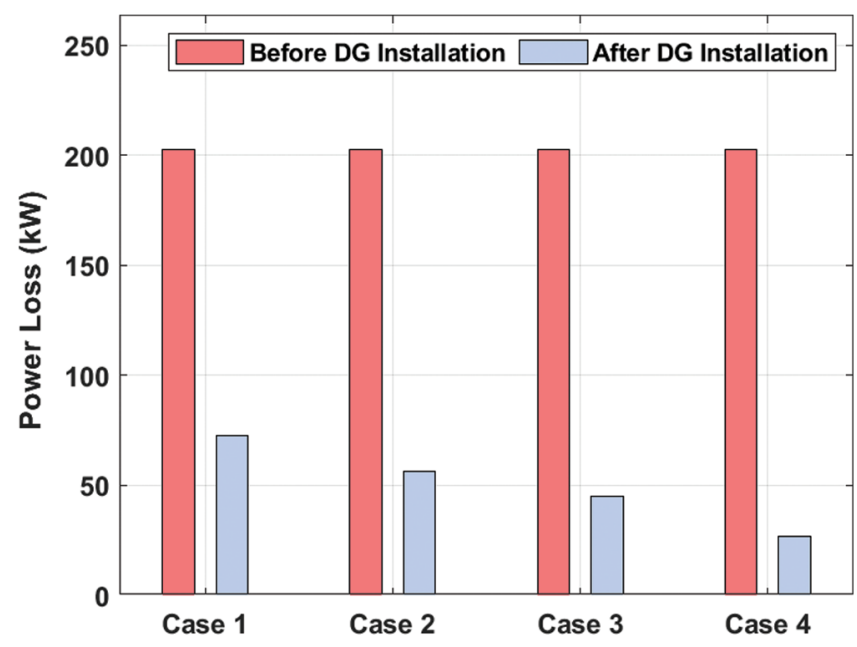

Figure 5: PL analysis of CAFO-OPSDG model on IEEE 33 bus system

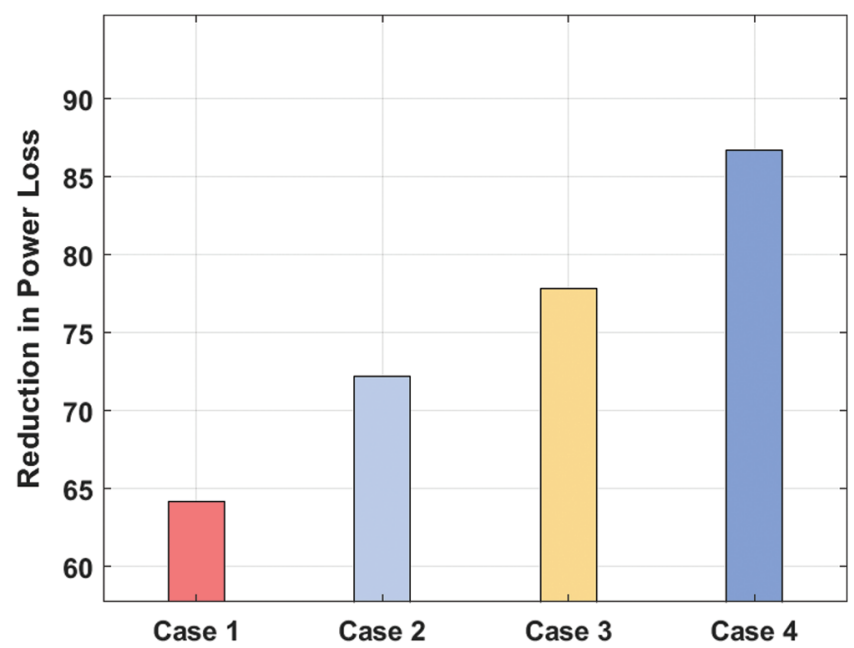

Figure 6: Reduction in PL analysis of CAFO-OPSDG model on IEEE 33 bus system

A comprehensive outcome analysis of the proposed CAFO-OPSDG approach on IEEE 69 bus system under distinct scenarios is provided in Tab. 2 and Figs. 7 and 8. From the table values, it is understood that the proposed CAFO-OPSDG technique accomplished minimal PL on all the applied cases. For instance, in case 1, the CAFO-OPSDG approach reduced the PL from 224.9804 to $131.4352 \mathrm{~kW}$, with $41.5793 \%$ reduction in PL. At the same time, the CAFO-OPSDG technique mitigated PL from 224.9804 to $140.0973 \mathrm{~kW}$, with a $37.7291 \%$ reduction. Moreover, in case 3, the CAFO-OPSDG technique outperformed other cases and reduced the PL from 224.9804 to $37.6289 \mathrm{~kW}$, with $83.2745 \%$ reduction. Furthermore, the CAFO-OPSDG model accomplished the least PL by reducing it from 224.9804 to $61.5368 \mathrm{~kW}$ i.e., $72.6479 \%$ reduction. From these values, it is evident that the presented CAFO-OPSDG technique has accomplished the least PL. Conversely, when investigating the performance with respect to minimized voltage, the CAFO-OPSDG technique produced a high minimized voltage. For instance, under case 1 , the CAFO-OPSDG approach obtained a minimized voltage of $0.95452 \mathrm{pu}$. 
Table 2: Comparison of the proposed CAFO-OPSDG on IEEE 69 bus system at different scenarios

\begin{tabular}{lll}
\hline Case 1 & Before DG installation & After DG installation \\
\hline PL & $224.9804 \mathrm{~kW}$ & $131.4352 \mathrm{~kW}$ \\
\% Reduction in PL & - & 41.5793 \\
Minimized voltage (pu) & 0.90919 & 0.95452 \\
Case 2 & & \\
PL & $224.9804 \mathrm{~kW}$ & $140.0973 \mathrm{~kW}$ \\
\% Reduction in PL & - & 37.7291 \\
Optimal DG Position: 9 & & \\
Case 3 & $224.9804 \mathrm{~kW}$ & \\
PL & - & $37.6289 \mathrm{~kW}$ \\
\% Reduction in PL & & 83.2745 \\
Optimal DG Position: 17 & & \\
Case 4 & $224.9804 \mathrm{~kW}$ & \\
PL & - & $61.5368 \mathrm{~kW}$ \\
\% Reduction in PL & & 72.6479 \\
Optimal DG Position: 11, 8, 33 & & \\
\hline
\end{tabular}

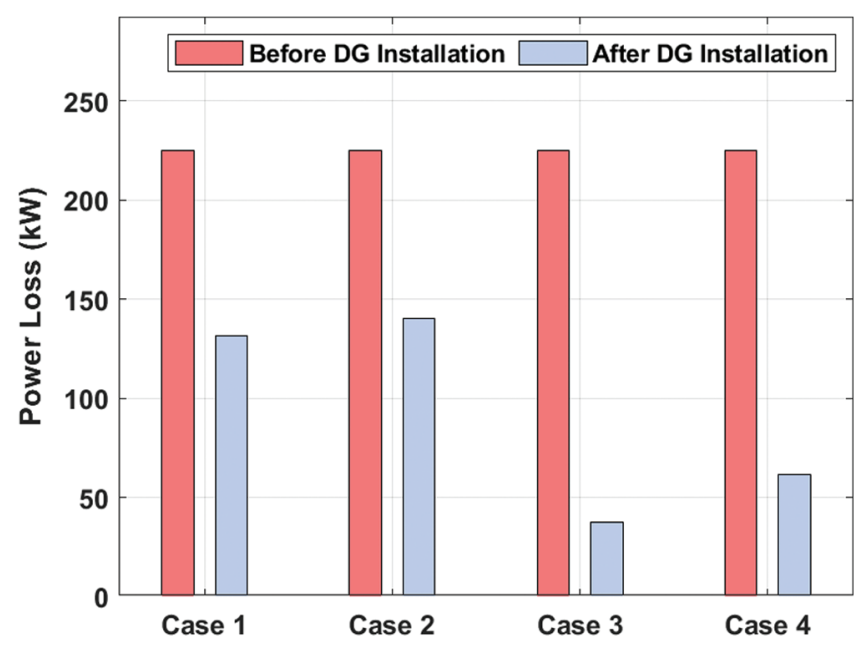

Figure 7: PL analysis of CAFO-OPSDG model on IEEE 69 bus system

Tab. 3 illustrates the results for comparative analysis of CAFO-OPSDG technique against existing techniques in terms of PL and loss reduction [19]. By examining the PL performance of the CAFOOPSDG technique against state-of-the-art methods, it is evident that the hybrid big bang technique offered the least performance over other methods with a PL of $139.530 \mathrm{~kW}$. In line with this, the Hybrid (HOAP) algorithm demonstrated a slightly-improved outcome with a PL of $81.05 \mathrm{~kW}$. Followed by, the hybrid (BPSO-SLFA) algorithm achieved a moderate PL of $31.284 \mathrm{~kW}$. Eventually, the hybrid (GWO) algorithm portrayed a competitive outcome with a PL of $28.862 \mathrm{~kW}$. Though the PSO-GA technique 
showcased better performance over earlier methods with a PL of $28.764 \mathrm{kWN}$, the proposed CAFO-OPSDG technique outperformed all other techniques by achieving a PL of $26.862 \mathrm{~kW}$.

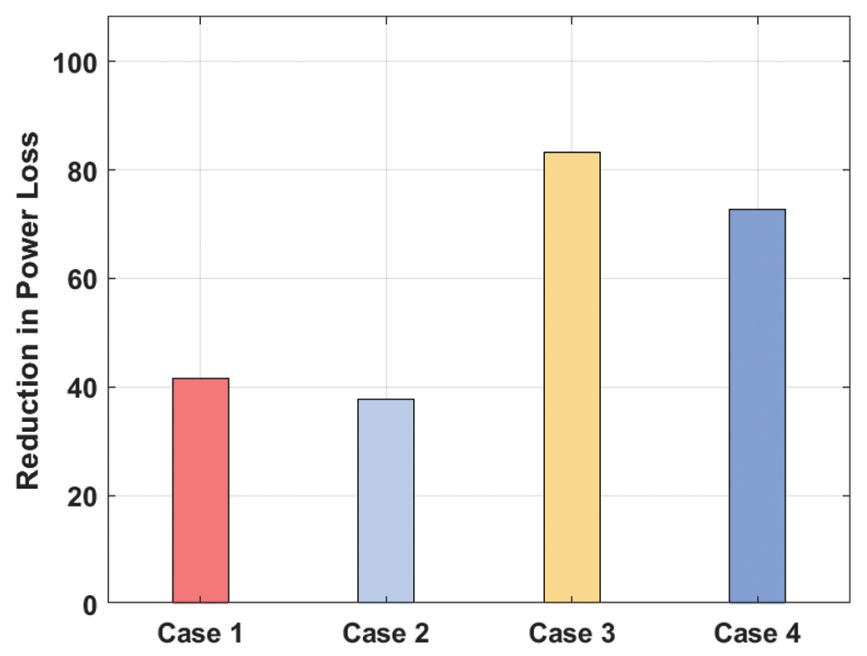

Figure 8: Reduction in PL analysis of CAFO-OPSDG model on IEEE 69 bus system

Table 3: Results of the existing models against the proposed model for allocation and sizing of multiple DGs

\begin{tabular}{lll}
\hline Methods & Power loss (kW) & Loss reduction $(\%)$ \\
\hline CAFO-OPSDG & 26.862 & 86.75 \\
PSO-GA & 28.764 & 85.80 \\
Hybrid (HAOP) & 81.050 & 61.62 \\
Hybrid (BPSO-SLFA) & 31.284 & 84.56 \\
Hybrid (HGWO) & 29.460 & 72.88 \\
Hybrid big bang & 139.530 & 78.73 \\
\hline
\end{tabular}

Fig. 9 examines the loss reduction performance of the proposed CAFO-OPSDG technique against stateof-the-art methods. From the figure, it is evident that the Hybrid (HAOP) technique offered poor performance over other methods with a loss reduction of $61.62 \%$. The performance of the Hybrid (HGWO) algorithm got certainly increased with a loss reduction of $72.88 \%$. Hybrid Big Bang algorithm resulted in a moderate loss reduction of $78.73 \%$. Moreover, the hybrid (GWO) algorithm portrayed a competitive outcome with a loss reduction of $84.56 \%$. In line with this, the PSO-GA technique exhibited a nearly acceptable performance with a loss reduction of $85.80 \%$, which was superior to all other methods, except the proposed CAFOOPSDG technique. In other terms, the proposed CAFO-OPSDG technique accomplished a superior performance with a loss reduction of $86.75 \%$. From the above mentioned results and discussion, it is apparent that the CAFO-OPSDG technique can be utilized as an appropriate tool for voltage profile enhancement and loss minimization in distribution networks. This is attributed to the fact that the CAFO algorithm incorporated the concept of chaos theory to improve the global optimization capabilities of traditional AFO algorithm. 


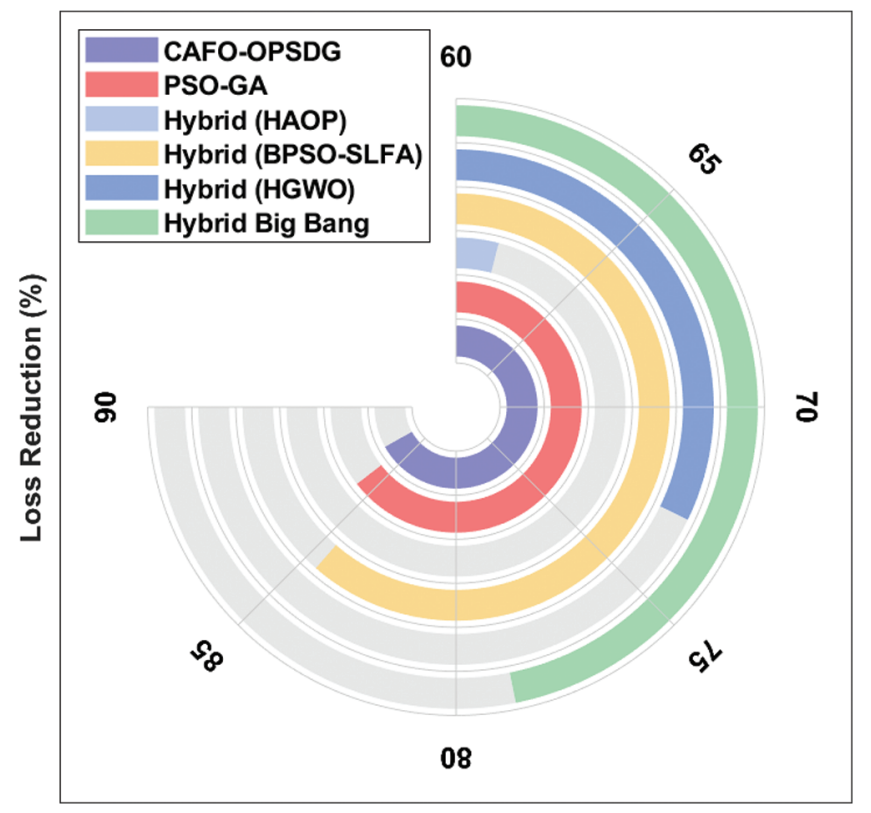

Figure 9: Loss reduction analysis of CAFO-OPSDG model

\section{Conclusion}

The current research study developed a novel CAFO-OPSDG algorithm to improve the voltage profiles and mitigate the PL. CAFO algorithm incorporated the concept of chaos theory to improve the global optimization capabilities of traditional AFO algorithm. The proposed CAFO-OPSDG algorithm was experimentally validated in IEEE 33 Bus system and IEEE 69 Bus system. The simulation results guaranteed the significant results of the proposed CAFO-OPSDG algorithm in terms of voltage deviation and boost-up voltage profile in power system. The proposed method decreased the power loss to $26.862 \mathrm{~kW}$ while the loss reduction was $86.75 \%$. Therefore, the CAFO-OPSDG technique can be employed as an effective model for optimum placement and sizing of DG units in distribution network systems. It exhibited promising results over the recent state-of-the-art methods. In future, the proposed CAFO-OPSDG technique can be extended to the design of short-term load prediction techniques to predict the forthcoming load as it helps in optimal resource allocation.

Funding Statement: The authors received no specific funding for this study.

Conflicts of Interest: The authors declare that they have no conflicts of interest to report regarding the present study.

\section{References}

[1] M. J. Tahir, I. A. Latiff, M. U. Gul, M. Alam and M. S. Mazliham, "Symmetrical and asymmetrical fault currents: Evaluation to enhance the performance of $220 \mathrm{KV}$ grid station," Journal of Telecommunication, Electronic and Computer Engineering, vol. 10, no. 1-3, pp. 147-152, 2018.

[2] M. Singh, V. Khadkikar, A. Chandra and R. K. Varma, "Grid interconnection of renewable energy sources at the distribution level with power-quality improvement features," IEEE Transactions on Power Delivery, vol. 26, no. 1, pp. 307-315, 2011.

[3] M. Delfanti, D. Falabretti and M. Merlo, "Dispersed generation impact on distribution network losses," Electric Power Systems Research, vol. 97, pp. 10-18, 2013. 
[4] U. Sultana, A. B. Khairuddin, M. M. Aman, A. S. Mokhtar and N. Zareen, "A review of optimum DG placement based on minimization of power losses and voltage stability enhancement of distribution system," Renewable and Sustainable Energy Reviews, vol. 63, no. 2, pp. 363-378, 2016.

[5] V. H. MendezQuezada, J. RivierAbbad and T. GomezSanRoman, "Assessment of energy distribution losses for increasing penetration of distributed generation," IEEE Transactions on Power Systems, vol. 21, no. 2, pp. 533540, 2006.

[6] A. K. Podder, S. Islam, N. M. Kumar, A. A. Chand, P. N. Rao et al., "Systematic categorization of optimization strategies for virtual power plants," Energies, vol. 13, no. 23, pp. 6251, 2020.

[7] J. Uthayakumar, N. Metawa, K. Shankar and S. K. Lakshmanaprabu, "Intelligent hybrid model for financial crisis prediction using machine learning techniques," Information Systems and e-Business Management, vol. 18, no. 4, pp. 617-645, 2020.

[8] S. K. Lakshmanaprabu, S. N. Mohanty, S. S. Rani, S. Krishnamoorthy, J. Uthayakumar et al., "Online clinical decision support system using optimal deep neural networks," Applied Soft Computing, vol. 81, pp. 105487, 2019.

[9] A. Naderipour, Z. Abdul-Malek, M. Hajivand, Z. M. Seifabad, M. A. Farsi et al., "Spotted hyena optimizer algorithm for capacitor allocation in radial distribution system with distributed generation (DG) and microgrid operation considering different load types," Scientific Reports, vol. 11, no. 1, pp. 1-15, 2021.

[10] W. Haider, S. J. U. Hassan, A. Mehdi, A. Hussain, G. O. M. Adjayeng et al., "Voltage profile enhancement and loss minimization using optimal placement and sizing of distributed generation in reconfigured network," Machines, vol. 9, no. 1, pp. 20, 2021.

[11] T. T. Nguyen and A. V. Truong, "Distribution network reconfiguration for power loss minimization and voltage profile improvement using cuckoo search algorithm," International Journal of Electrical Power \& Energy Systems, vol. 68, no. 1, pp. 233-242, 2015.

[12] S. Kasi and R. Neela, "Harris hawks optimization algorithm based power loss minimization in micro grid incorporated with distributed generation," IOP Conference Series: Materials Science and Engineering, vol. 1070, pp. 12099, 2021.

[13] S. Essallah and A. Khedher, "Optimization of distribution system operation by network reconfiguration and DG integration using MPSO algorithm," Renewable Energy Focus, vol. 34, no. 4, pp. 37-46, 2020.

[14] S. P. Dash, K. R. Subhashini and J. K. Satapathy, "Optimal location and parametric settings of FACTS devices based on JAYA blended moth flame optimization for transmission loss minimization in power systems," Microsystem Technologies, vol. 26, no. 5, pp. 1543-1552, 2020.

[15] A. Ramamoorthy and R. Ramachandran, "Optimal siting and sizing of multiple DG units for the enhancement of voltage profile and loss minimization in transmission systems using nature inspired algorithms," The Scientific World Journal, vol. 2016, no. 2, pp. 1-16, 2016.

[16] M. G. Yenealem, L. M. H. Ngoo, D. Shiferaw and P. Hinga, "Management of voltage profile and power loss minimization in a grid-connected microgrid system using fuzzy-based STATCOM controller," Journal of Electrical and Computer Engineering, vol. 2020, no. 5, pp. 1-13, 2020.

[17] I. Ziari, G. Ledwich, A. Ghosh, D. Cornforth and M. Wishart, "Optimal allocation and sizing of capacitors to minimize the transmission line loss and to improve the voltage profile," Computers \& Mathematics with Applications, vol. 60, no. 4, pp. 1003-1013, 2010.

[18] K. R. Devabalaji and K. Ravi, "Optimal size and siting of multiple DG and DSTATCOM in radial distribution system using bacterial foraging optimization algorithm," Ain Shams Engineering Journal, vol. 7, no. 3, pp. 959-971, 2016.

[19] A. S. Hassan, Y. Sun and Z. Wang, "Multi-objective for optimal placement and sizing DG units in reducing loss of power and enhancing voltage profile using BPSO-SLFA," Energy Reports, vol. 6, no. 4, pp. 1581-1589, 2020.

[20] L. Cheng, X. Wu and Y. Wang, "Artificial flora (AF) optimization algorithm," Applied Sciences, vol. 8, no. 3, pp. $329,2018$.

[21] I. Strumberger, E. Tuba, N. Bacanin, M. Zivkovic, M. Beko et al., "Designing convolutional neural network architecture by the firefly algorithm," in 2019 International Young Engineers Forum (YEF-ECE), Costa da Caparica, Portugal, pp. 59-65, 2019. 
[22] O. S. Qasim, N. A. Al-Thanoon and Z. Y. Algamal, "Feature selection based on chaotic binary black hole algorithm for data classification," Chemometrics and Intelligent Laboratory Systems, vol. 204, pp. 104104, 2020.

[23] B. B. Zad, H. Hasanvand, J. Lobry and F. Vallée, "Optimal reactive power control of DGs for voltage regulation of MV distribution systems using sensitivity analysis method and PSO algorithm," International Journal of Electrical Power \& Energy Systems, vol. 68, no. 3, pp. 52-60, 2015. 